Instituto Internacional de Investigación y Desarrollo Tecnológico Educativo INDTEC, C.A.

DOI: https://doi.org/10.29394/scientific.issn.2542-2987.2017.2.3.19.343-360

OAI-PMH: http://www.indteca.com/ojs/index.php/Revista Scientific/oai

\title{
Capacitación en Valores para Fortalecer la Convivencia Social y Ciudadana
}

Autora: Yajaira del Valle Cadenas Terán

Universidad Nacional Experimental Rafael María Baralt, UNERMB

yajairaf@yahoo.com

Trujillo, Venezuela

Resumen

El propósito de este estudio es exponer de forma significativa la capacitación en valores para fortalecer la convivencia social y ciudadana tomando en cuenta que la convivencia del ser humano no ha sido del todo fácil, pero es hasta ahora que se han logrado relacionar muchos de sus problemas con la forma de relacionarse y de interactuar con el medio ambiente, sobre todo con el medio social. Este estudio se realizó con una revisión bibliográfica que sirve de fundamento para la capacitación dirigida al fortalecimiento de la convivencia y ciudadanía, por tanto se enmarca desde una investigación de tipo descriptivo-documental. Concluyendo, con la importancia de la temática dado que se abran puertas para los valores, diálogos, reflexiones, calidad de vida, entre otros que reflejen el accionar del docente basado en principios axiológicos.

Palabras clave: valores; convivencia social; ciudadanía. 


\title{
Training in Values to Strengthen Social and Civic Coexistence
}

\begin{abstract}
The purpose of this study is to present significantly values training to strengthen social and citizen considering that the coexistence of human beings has not been entirely easy, but so far they have managed to relate many of their problems with how to relate and interact with the environment, especially with the social environment. This study was conducted with a literature review which is the basis for targeted strengthening coexistence and citizenship therefore falls from a descriptive research training documentary. In conclusion, the importance of the subject since doors for values, dialogues, reflections, quality of life, among others that reflect the actions of teachers based on principles axiological open.
\end{abstract}

Keywords: values; social affairs; citizenship. 


\section{Introducción}

El ser humano no puede vivir aislado de los demás y, a pesar de definirse como el ser más perfecto de la naturaleza, tiene carencias que lo limitan y lo hacen vulnerable y sensible a los efectos del ambiente natural, físico y social que lo rodea. Es un ser carencial, porque no cuenta con muchas armas naturales que le permitan sobrevivir en cualquier lugar y medio. Pero también es un ser en evolución constante. Su desarrollo no sólo es cronológico, sino que evoluciona en su pensamiento, capacidad de conocer, de aprender, de sentir, de expresarse, de comunicarse y de adaptar su entorno a sus necesidades inmediatas.

Por lo tanto, se vive en una sociedad donde los valores escasean, están de baja. Se oye decir mucho: “¡Qué más da! ¡Todo da igual!”. Este encogerse de hombros y de energías, singular reflejo de una crisis de vida, de una época de desaliento, desencanto, confusión, promesas incumplidas, de falta de horizonte, parece que tiene una causa: "ausencia de valores".

Por ello, se buscó el apoyo en lo que dice, Arévalo (1998), define el valor como: "todo aquello que favorece la plena realización de nosotros como persona" (pág. 65). Ello justifica, en primer lugar, en el orden de prioridades y configuración de lo más íntimo de cada individuo. De acuerdo con esta postura, es de considerar que el concepto de valor en la actualidad, debido a los múltiples y frenéticos cambios sociales, motivados unas veces por la mayor o menor influencia política y económica de los diferentes países, han dado lugar a un sistema de valores cuyos principales determinantes son la competitividad y el individualismo.

Por esto, la formación de valores enseña a tener aprecio por las cosas que satisfacen las necesidades básicas, pero se valora especialmente a las personas que las proporcionan. Su comportamiento hacia los demás se vuelve la principal referencia de lo que es valioso. Por esta razón, el carácter y personalidad se moldea con las actitudes, comportamientos de las personas 
que nos crían, bien sea los padres u otros familiares. Sus conductas tienen el principal peso de lo que después se convierte en nuestros principios y creencias personales más importantes.

Por otro lado, la convivencia es la condición de relacionarse con las demás personas o grupos a través de una comunicación permanente fundamentada en afecto y tolerancia que permite convivir y compartir en armonía en las diferentes situaciones de la vida. La convivencia social consiste en el respeto mutuo entre las personas, las cosas y el medio en el cual vivimos y desarrollamos nuestra actividad diaria. De ahí, la importancia de las leyes porque éstas regulan y garantizan el cumplimiento de esa convivencia social.

De hecho, en el Artículo 1 de la Constitución de la República Bolivariana de Venezuela (1999), se expresa que: "Venezuela se declara República Bolivariana, irrevocablemente libre $e$ independiente y fundamenta su patrimonio moral, así como los valores de libertad, igualdad, justicia, y paz internacional, en la doctrina de Simón Bolívar, el Libertador...". Asimismo, según García (2002):

La ética y la ciudadanía van de la mano, puesto que la ética establece las normas de conducta que deben regir a los ciudadanos de una región y ellos son los responsables de aplicar y cumplir con ética las distintas reglas y normas que se establezcan para una determinada región (pág. 65).

Por los planteamientos hechos es importante cumplir con el objetivo de caracterizar la capacitación en Valores para fortalecer la convivencia social y ciudadana para vincular, los nuevos Programas Nacionales de Formación (PNF), que se llevan a cabo en las universidades del país cumpliendo para esto con una metodología de tipo documental, descriptiva porque las fuentes consultadas son propias para dejar clara la convivencia social y ciudadanía. 
De forma concluyente, educar para la formación en valores para una sana convivencia social y ciudadana presume apostar por modelos pedagógicos, en el cual se procure que la persona construya su propio proyecto de vida y al mismo tiempo, contribuya a la construcción de una sociedad justa y democrática, donde la convivencia refleje que se pretende construir una ciudadanía, y sobre todo, sí ésta se manifiesta en sociedades plurales y diversas.

\section{Planteamiento del Problema}

El hombre de hoy, a nivel mundial, presenta un gran vacío de valores, los cambios sociales y económicos implica un reflejo de alteración en éstos donde predomina el dinero y el amor a los bienes materiales, motivo por el cual se producen distanciamientos en los núcleos familiares. Así, las causas más comunes en la pérdida de valores son: la desintegración y los conflictos en las familias, los divorcios, la situación económica, deserción escolar, desobediencia, el alcoholismo y la drogadicción. Esto, trae como consecuencia, según Keith y Newstrom (2000): "el surgimiento de bandas delictivas, prostitución, embarazos precoces, robos, transculturación, abortos en jóvenes, sexualidad prematura" (pág. 21).

No obstante, la principal educación nace y se desarrolla en el hogar. Es ahí donde aprende a despertar interés por la vida, a confiar en sí mismo, a creer que puede seguir adelante por los caminos del triunfo. La familia es, en su más profundo sentido, comunidad y comunicación. Es la primera comunidad de vida de amor, pero es al mismo tiempo la primera escuela del saber, del civismo y de la ciudadanía. Es la primera escuela de los hijos. La familia es la sociedad creada para educar generaciones. Unos padres que sepan educar, son seres que planifican para tener a sus hijos, que se preparan para esta tarea tan sublime de saber ser padre. 
Es de resaltar, que esta situación, es un problema de familia disfuncional, pero hoy en día, se ha convertido en una preocupación del Estado Venezolano, porque está afectado seriamente la convivencia social y ciudadana de la colectividad, lo que ocasiona notablemente en cualquier rincón la violencia, ya que cada día se están formando más grupos de pandillas, que provocan actos delictivos, como: inseguridad, drogadicción, entre otros.

Por lo tanto, la Constitución de la República Bolivariana de Venezuela (1999): enfatiza que el gobierno debe garantizar la convivencia de los diversos grupos sociales sin que se produzcan estallidos de violencia. Para dar cumplimiento a este criterio, el gobierno revolucionario y socialista ha creado leyes y ordenanzas según cada comunidad específica, sobre convivencia social y ciudadana para el buen vivir.

Según, Cárdenas (2010), dice que: "la falta de convivencia en la sociedad se refiere a los comportamientos que no cumplen las reglas y normas establecidas dentro del control y el orden" (pág. 11). Entre las causas más importantes que se encuentran la falta de motivación, la baja autoestima, un nivel de desarrollo cognitivo insuficiente para el autocontrol que el sistema disciplinario exige, inestabilidad familiar, y poca calidad del entorno en el que viven los individuos. Por otro lado, otras de las causas de estas situaciones, pueden ser la falta de conocimiento y preparación de los padres en este tema, lo cual hace que ellos manejen la situación de una manera superficial, en lugar de hacerlo a profundidad y de una forma adecuada que resuelva el problema definitivamente y no por un momento.

En este sentido, la convivencia permite al ser humano vivir de acuerdo con las reglas de la sociedad, así también él puede obtener la aprobación y armonía con los miembros, es una motivación porque ayuda a desarrollar su personalidad. En otras palabras, una buena convivencia es sinónimo de educación y orientación, de formación de valores, así como fortalecimiento de 
valores porque enfatiza en el crecimiento integral, la autodisciplina, el autocontrol y al mismo tiempo guía la motivación para lograr llegar a obtener los objetivos sin problemas para crear su felicidad y una buena adaptación personal y social.

Por los argumentos explicados anteriormente es que se pone en práctica esta investigación, para vincular, los nuevos Programas Nacionales de Formación (PNF), que se llevan a cabo en las universidades del país con todos los sectores que conforman el ámbito universitario, las comunidades y los distintos entes de Estado. Según, el Ministerio del Poder Popular para la Educación Superior (2008), el PNF se busca con la construcción de:

Una cultura social incluyente, dentro de un modelo productivo socialista, humanista, endógeno, arraigado en las tradiciones de lucha del pueblo y en la voluntad política del gobierno de avanzar hacia la creación de un escenario social que permita alcanzar la suprema felicidad del ser humano (pág. 40).

Por ende, este estudio busca dar respuesta a la siguiente interrogante ¿Cómo determinar los nuevos Programas Nacionales de Formación (PNF), que se llevan a cabo en las universidades del país con todos los sectores que conforman el ámbito universitario?

\subsection{Bases Teóricas}

\subsubsection{Valores}

La formación de valores enseña a tener aprecio por las cosas que satisfacen las necesidades básicas, pero se valora especialmente a las personas que las proporcionan. Su comportamiento hacia los demás se vuelve la principal referencia de lo que es valioso. Por esta razón, el carácter y personalidad se moldea con las actitudes y comportamientos de las personas que nos crían, bien sea los padres u otros familiares. Sus conductas tienen el principal peso de lo que después se convierte en nuestros principios y 
creencias personales más importantes.

Los valores son principios que permiten orientar el comportamiento en función de la realización como personas. Son creencias fundamentales que ayudan a preferir, apreciar, elegir unas cosas en lugar de otras, o un comportamiento en lugar de otro. También, son fuente de satisfacción y plenitud. Proporcionan una pauta para formular metas y propósitos, personales o colectivos. Reflejan intereses, sentimientos y convicciones más importantes. Los valores se refieren a necesidades humanas y representan ideales, sueños y aspiraciones, con una importancia independiente de las circunstancias.

Educar para la formación en valores para una convivencia social y ciudadana supone apostar por un modelo pedagógico, en el cual se procure que la persona construya su propio proyecto de vida y al mismo tiempo, apoye a la construcción de una sociedad justa y demócrata. Esta doble distancia individual, relacional, particular, social, debe enlazar en el mismo tiempo y espacio si lo que se pretende es construir ciudadanía y sobre todo, sí ésta se manifiesta en sociedades plurales con diversidad.

Ahora bien, partiendo del concepto de valor, y establecidas las bases sobre las que debe cimentarse la educación en valores de acuerdo a lo que diseña el Ministerio de Educación y Deporte (1997): en el marco del Currículo Básico Nacional, la educación en valores, constituye, en primer lugar, uno de los compendios y objetivos esenciales de la educación en los cuales se establece:

Adecuar el sistema educativo al proceso de cambio y a las transformaciones profesionales que se están generando en todas las estructuras del país, ampliando los niveles de participación de todos los actores a la formación mínima y obligatoria de la población. Determinar el perfil de opciones de los distintos actores del sistema educativo y de la sociedad civil en relación con las decisiones que afectan a los diferentes componentes que interactúan a nivel educativo (pág. 27). 
O sea, en el contexto de esos objetivos respondiendo al reto que presumen en el ámbito escolar, en el currículo se plantea la educación en los valores como un eje transversal, es decir, como una extensión educativa global interdisciplinaria que empapa todas las áreas y que se desarrolla transversalmente en todos los componentes del currículo. No obstante, se considera que el reto que admite la atención de valores, implica una responsabilidad de la sociedad educativa para convertir la globalidad del proceso de enseñanza y aprendizaje en un proceso efectivamente humanizador.

Por tanto, la presencia de los valores en el diseño curricular, se justifica por la crisis moral que identifica la época actual. Sin embargo, una educación en valores debe suscitar cambios reveladores que lleven a la formación de un ser humano capaz de desenvolverse en una sociedad pluralista en la que pueda, de una manera crítica y reflexiva, practicar como norma de vida la libertad, convivencia, tolerancia, solidaridad, honestidad y justicia. Visto desde el ámbito educativo, surge el respeto de la educación en los valores, dentro del currículo, como un proceso o, si se quiere, como un adjunto de enseñanza y aprendizaje que debe empapar la totalidad de la tarea educativa.

Así lo explica, Sebastiao (2004): "en las organizaciones, los valores permiten que sus integrantes interactúen de manera armónica. Influyen en su formación y desarrollo como personas, y facilitan alcanzar objetivos que no serían posibles de manera individual" (pág. 231). O sea, para el bienestar de una comunidad es necesario que existan normas compartidas que orienten el comportamiento de sus integrantes.

Asimismo, Hernández (2003), dice que: "para la cultura organizacional de una empresa los valores son la base de las actitudes, motivaciones y expectativas de sus trabajadores" (pág. 28). O lo que es lo mismo, los valores son la columna vertebral de sus comportamientos. Si los valores no tienen significados comunes para todos los empleados, el trabajo diario se hace más 
difícil y pesado. El ambiente laboral se vuelve tenso, la gente trabaja con la sensación de que no todos reman en la misma dirección y los clientes pagan las consecuencias. Como pilares de una empresa, los valores no sólo necesitan ser definidos, la empresa debe darles mantenimiento, promoverlos y divulgarlos constantemente. Sólo así sus trabajadores tendrán mejor oportunidad de comprender sus significados y ponerlos en práctica en sus labores diarias.

\subsubsection{Tipos de Valores}

En este sentido, es importante destacar, según Jiménez (2008: 37), los tipos de valores:

(1) Valores espirituales: Se refieren a la importancia que le damos a los aspectos no-materiales de nuestras vidas. Son parte de nuestras necesidades humanas y nos permiten sentirnos realizados. Le agregan sentido y fundamento a nuestras vidas, como ocurre con las creencias religiosas.

(2) Valores morales o humanos: Son las actitudes y conductas que una determinada sociedad considera indispensables para la convivencia, el orden y el bien general.

(3) Valores personales: Son aquellos que consideramos principios indispensables sobre los cuales construimos nuestra vida y nos guían para relacionarnos con otras personas. Por lo general son una mezcla de valores familiares y valores socio-culturales, juntos a los que se agrega como individuos según nuestras vivencias.

(4) Valores sociales: Son los que imperan en la sociedad en la que vivimos. Han cambiado a lo largo de la historia y pueden coincidir o no con los valores familiares o los personales. Se trata de una mezcla compleja de distintos tipos de valoraciones, que en muchos casos parecen contrapuestas o plantean dilemas.

(5) Valores materiales: Son aquellos que nos permiten subsistir. Tienen 
que ver con nuestras necesidades básicas como seres humanos, como alimentarnos o vestirnos para protegernos de la intemperie. Son importantes en la medida que son necesarios. Son parte del complejo tejido que se forma de la relación entre valores personales, familiares y socio-culturales. Cuando se exageran, los valores materiales entran en contradicción con los espirituales. En este orden de ideas, cuando en la familia, la escuela, el trabajo, y en la sociedad en general, hay fallas de funcionamiento, muchas veces se debe a la falta de valores compartidos, lo que se refleja en falta de coherencia entre lo que se dice y lo que se hace.

La ética sirve para vivir auténticamente mejor, para ser mejores personas, por lo que, fuera del contexto ejemplarizante de esta ciencia aplicada, se evidencia en la sociedad actual, un franco deterioro social, familiar, económico, político, cultural, asistencial, por el cual atraviesa Venezuela, observado en actitudes típicas de una sociedad en crisis, expresadas, que según Bortone (1998): "en ambientes familiares inestables, pérdidas de las costumbres ciudadanas, poco amor hacia el trabajo, irrespeto a la dignidad de las personas, y en general, existe una marcada inclinación, hacia actitudes tendientes a tener más que al ser" (pág. 32).

\subsection{Convivencia Social y Ciudadana}

Cuando se habla de una convivencia social y ciudadana, debemos ubicarnos en el espacio y tiempo, tomando en cuenta los diferentes escenarios que constituyen nuestra organización o institución, en este caso el educativo. En este momento, contamos con notables avances en la conciencia moral de la sociedad respecto, entre otros, del derecho a la vida, a la libertad, al trabajo, a la educación, a la salud, a la tierra, al medio ambiente, así como los derechos de los niños, ancianos, mujeres, las personas con necesidades especiales, indígenas, inmigrantes y grupos sociales 0 sectores que sufren 
discriminaciones particulares.

Por esto, una ciudadanía social y responsable, para Yslado (2003), es la que:

En la sociedad actual, la educación debe contribuir a formar personas que puedan convivir en un clima de respeto, tolerancia, participación y libertad y que sean capaces de construir una concepción de la realidad que integre a la vez el conocimiento y la valoración ética y moral de la misma (pág. 89).

Esta concepción cívica y humanista de la educación es la que propugna la Constitución Nacional de la República Bolivariana (1999), así como la Ley Orgánica de Educación (2009): la formación en el respeto de los derechos, libertades fundamentales, en el ejercicio de la tolerancia y de la libertad.

Analizando estas bases fundamentales y legales, es importante reflexionar acerca del espíritu de cada una de ellas, que toman en cuenta como objetivo primero y fundamental de la educación proporcionar a estudiantes una conceptualización de la realidad capaz de integrar el conocimiento y la valoración ética y moral de la misma mediante la transmisión, ejercicio de los valores que hacen posible la vida en sociedad, el respeto a derechos y libertades fundamentales.

Cuando se trata de dar una conceptualización clara de lo que significa una convivencia social, sostiene Guerra (2004): "hay que tomar en cuenta diversos documentos y declaraciones internacionales" (pág. 21); es decir, se refieren a la escuela como promotora de la convivencia social y ciudadana activa y de la cohesión social a través de la enseñanza que realiza de los valores cívicos.

Considerando, que al hablar de ciudadanía, hay que tomar en cuenta lo que significa ser ciudadano, por cuanto éste es miembro de una comunidad política, y que su condición de miembro se conoce como ciudadanía, lo que conlleva a una serie de derechos y deberes que tiene que asumir. Surge una 
interrogante, ¿cómo definir ciudadanía? Quizás esta definición dada por Escámez (2006): reviste filosóficamente el espíritu de la misma cuando sostiene que "es el derecho y la disposición de participar en una comunidad, a través de la acción autorregulada, exclusiva, pacífica y responsable, con el objeto de optimizar el bienestar público" (pág. 89).

Ante esta realidad, el rol del docente en la educación es conducir al ser humano hacia su realidad social y ayudarlo a interpretarla, para que asuma la responsabilidad de sus actos En el marco de generar este tipo de ciudadano se requiere facilitar el aprendizaje verdadero, lo cual depende de ciertas cualidades en las actitudes de relación personal entre el educador, la familia, la sociedad y el que aprende. La educación para la formación de actitudes es una tarea más interesante que la simple transmisión de los contenidos curriculares, y no porque los contenidos se olvidan, sino porque en lo moral, la actitud, la predisposición del que obra, sigue siendo una pieza clave para la vida.

\section{Metodología}

Para Bavaresco (2006): hablar de investigación es referirse a "una manera planificada, cautelosa, sistemática y confiable de descubrir o profundizar el conocimiento" (pág. 24). De allí, que el presente estudio, es de carácter documental o bibliográfico. En tal sentido, Tamayo y Tamayo (2004), señala que:

El tipo de investigación se determina de acuerdo con el tipo de problema que el investigador deseó solucionar, los objetivos que éste pretendió lograr y de la disponibilidad de todos los recursos que se necesiten para la culminación del estudio (pág. $67)$.

Razón por la cual, se afirma que el presente estudio, es de carácter documental y de campo, por lo que los datos se recabaron por medio de 
fuentes directas e indirectas, como; documentos de diversa índole, elaborados y procesados con anterioridad al trabajo. Por su parte, Sabino (2005), expresa que: "la investigación documental constituye un procedimiento científico sistemático, de indagación, recolección, organización, interpretación y presentación de datos e información alrededor de una estrategia de análisis de documentos" (pág. 37). Además, Bernal (2000): señala que los estudios documentales "consisten en un análisis de la información sobre un determinado tema, con el propósito de establecer relaciones, diferencias, etapas, posturas o estado actual del conocimiento, respecto al tema objeto de estudio" (pág. 52).

\section{Consideraciones Finales}

A continuación, se muestran las consideraciones finales a las que se concluyó luego de realizar el estudio:

Los compendios relacionados con la formación en valores para una convivencia social y ciudadana convergen en un momento dado con la necesidad de pensar en la educación como un proceso social e integrador, impregnada de valores. la sociedad actual debe contribuir a formar ciudadanos que puedan convivir en las organizaciones con el respeto, la tolerancia, participación y libertad, capaces de construir una concepción de la realidad contextual.

Asimismo, el ciudadano debe estar formado en valores con una gran responsabilidad de manera integral para cumplir con las normas que la sociedad le está exigiendo; sin embargo, sería interesante, que este mismo concepto lo internalice el docente como gestor de su praxis valorativa. Para así, tomar acciones que deriven en esfuerzos comunes para colocar en nuevos escenarios cambios significativos, diferentes actitudes, espacios de aprendizajes imborrables y de valores, con el fin de aprovechar las ventajas como calificativo para reflexionar, la comunicación y una socialización 
focalizada en un sentido humanista.

Al abordar el tema de la capacitación en valores para fortalecer la convivencia social y ciudadana, cabría preguntarse si es necesaria esta capacitación, ya que es posible pensar que no se necesitan habilidades ni estrategias particulares para educar en valores y convivencia social y ciudadana, sino que este es un proceso que se da de manera natural en el diario quehacer educativo; sin embargo, también considero que hay que prestarle atención a la escuela porque es el espacio social y privilegiado para fomentar el pensamiento, el comportamiento valorativo, y para iniciar a practicar la ciudadanía a través de la convivencia.

En síntesis, en este momento asumo que al destronar de actitudes que afecten el camino para transitar para la formación en valores y tener una convivencia social y ciudadana, estaremos abriendo una salida en donde las actitudes, experiencias, confianza, imagen docente, humanización, diálogos valorativos, reflexión, comunicación, calidad de vida, entorno social y los diferentes cambios, serán reflejo de una convivencia comprometida con el activo humano que realiza su función como docente.

\section{Referencias}

Arévalo, M. (1998). La Educación Social para una Escuela Competitiva. Ediciones Romar. Argentina.

Bavaresco, A. (2006). Técnicas de investigación. Maracaibo. Editorial Universidad del Zulia, LUZ.

Bernal, C. A. (2000). Metodología de la investigación para administración y economía. Prentice Hall. Madrid.

Bortone Di Muro, R. (1998). Resultados de la Aplicación de un Programa de Desarrollo Personal dirigido a Estudiantes de la Carrera Ingeniería Electrónica-UNET. Trabajo de ascenso no publicado. Universidad Nacional Experimental del Táchira. 
Cárdenas, G. (2010). La Disciplina, Autonomía y Desarrollo Moral. Artículo publicado en la Revista Xictli de la Unidad UPN 094 D.F. Centro, México. Recuperado de: http://u094.upnvirtual.edu.mx

Constitución de la República Bolivariana de Venezuela (1999). Gaceta Oficial № 36.860. Caracas, Venezuela: Asamblea Nacional Constituyente.

Escámez, J. (2006). Los Valores para la Educación en la ciudadanía en el contexto familiar. Universidad de Valencia. Recuperado de: http://consumoetico.webs.uvigo.es/textos/conversas pedagoxicas/valo res y ciudadania en el contexto familiar.doc

García, J. (2002). Ética y ciudadanía. Recuperado de: https://www.monografias.com

Guerra, G. (2004). Ética y Axiología en una Sociedad Libre de Conflictos. Editorial Panapo. Caracas, Venezuela.

Hernández, M. (2003). Los valores de la sociedad. s.e.

Jiménez, J. (2008). El valor de los valores en las organizaciones. $1^{\text {a }}$. Edición. Caracas, Venezuela: Cograf Comunicaciones.

Keith, D., y Newsroom, J. (2000). Comportamiento Humano en Trabajo. México: McGraw-Hill.

Ley Orgánica de Educación (2009). Gaceta Oficial N 5.929 Extraordinario del 15 de agosto. República Bolivariana de Venezuela: La Asamblea Nacional.

Ministerio de Educación y Deporte (1997). Dentro del Currículo Básico Nacional. Caracas, Venezuela.

Ministerio del Poder Popular para la Educación Superior (2008). Programa Nacional de Formación. Caracas, Venezuela: Autor.

Sabino, C. (2005). Metodología, diseño y desarrollo del proceso de investigación. Colombia: McGraw-Hill.

Sebastiao, B. (2004). Aproximación al concepto del Derecho desde la Perspectiva Tríadica. Tesis doctoral. [Documento en línea]. 
Recuperado de: http://www.eumed.net/tesis/sb

Tamayo y Tamayo, M. (2004). El Proceso de Investigación Científica.

México, DF.: Limusa Noriega Editores.

Yslado, R. (2003). Educación en valores. [CD Room]. Trujillo, Venezuela. 


\section{Yajaira del Valle Cadenas Terán}

\section{e-mail: sonia aguilar1965@hotmail.com}

Nacida en Valera, estado Trujillo, Venezuela.

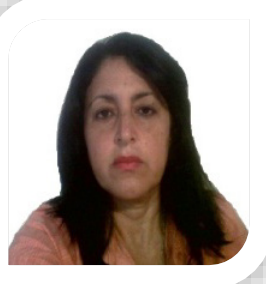

Actualmente cursa estudios de Doctorado en Ciencias de la Educación en la Universidad Nacional Experimental "Rafael María Baralt". Maestría en Administración de la Educación Básica Magíster Scientiarum 1994-1997; Diplomado en Cooperativismo y Gestión Comunitaria 2007 en el Instituto Universitario de Tecnología del Estado Trujillo; Curso de: Didáctica Especial en el Área de Castellano y Literatura, Matemática y Física y en el Área de Biología y Química 160 Horas 2010 (Diplomado) en el Liceo Bolivariano Antonio José Pacheco Estado Trujillo, convenio Cuba Venezuela. Experiencia Laboral actualmente Prof. Agregado de la Universidad Politécnica Territorial "Mario Briceño Iragorry".

El contenido de este manuscrito se difunde bajo una Licencia de Creative Commons ReconocimientoNoComercial-Compartirlgual 4.0 Internacional 